\title{
Acute Exercise-Induced Response of Monocyte Subtypes in Chronic Heart and Renal Failure
}

\author{
Amaryllis H. Van Craenenbroeck, ${ }^{1,2,3}$ Katrijn Van Ackeren, ${ }^{4}$ \\ Vicky Y. Hoymans, ${ }^{1,4}$ Johan Roeykens, ${ }^{5}$ Gert A. Verpooten, ${ }^{2,3}$ Christiaan J. Vrints, ${ }^{1,4,6}$ \\ Marie M. Couttenye, ${ }^{2,3}$ and Emeline M. Van Craenenbroeck ${ }^{1,4,6}$ \\ ${ }^{1}$ Laboratory of Cellular and Molecular Cardiology, Antwerp University Hospital, 2650 Antwerp, Belgium \\ ${ }^{2}$ Department of Nephrology, Antwerp University Hospital, 2650 Antwerp, Belgium \\ ${ }^{3}$ Laboratory of Experimental Medicine and Pediatrics, University of Antwerp, 2650 Antwerp, Belgium \\ ${ }^{4}$ Cardiovascular Diseases, Department of Translational Pathophysiological Research, University of Antwerp, 2650 Antwerp, Belgium \\ ${ }^{5}$ S.P.O.R.T.S., Antwerp University Hospital, 2650 Antwerp, Belgium \\ ${ }^{6}$ Department of Cardiology, Antwerp University Hospital, 2650 Antwerp, Belgium
}

Correspondence should be addressed to Amaryllis H. Van Craenenbroeck; amaryllis.vancraenenbroeck@uantwerpen.be

Received 12 September 2014; Revised 29 November 2014; Accepted 1 December 2014; Published 22 December 2014

Academic Editor: Eduardo López-Collazo

Copyright (C) 2014 Amaryllis H. Van Craenenbroeck et al. This is an open access article distributed under the Creative Commons Attribution License, which permits unrestricted use, distribution, and reproduction in any medium, provided the original work is properly cited.

\begin{abstract}
Purpose. Monocytes (Mon1-2-3) play a substantial role in low-grade inflammation associated with high cardiovascular morbidity and mortality of patients with chronic kidney disease (CKD) and chronic heart failure (CHF). The effect of an acute exercise bout on monocyte subsets in the setting of systemic inflammation is currently unknown. This study aims (1) to evaluate baseline distribution of monocyte subsets in CHF and CKD versus healthy subjects (HS) and (2) to evaluate the effect of an acute exercise bout. Exerciseinduced IL-6 and MCP-1 release are related to the Mon1-2-3 response. Methods. Twenty CHF patients, 20 CKD patients, and 15 HS were included. Before and after a maximal cardiopulmonary exercise test, monocyte subsets were quantified by flow cytometry: $\mathrm{CD}_{14}{ }^{++} \mathrm{CD} 16^{-} \mathrm{CCR}^{+}$(Mon1), $\mathrm{CD} 14^{++} \mathrm{CD}_{16}{ }^{+} \mathrm{CCR} 2^{+}$(Mon2), and CD14 ${ }^{+} \mathrm{CD} 16^{++} \mathrm{CCR}^{-}$(Mon3). Serum levels of IL-6 and MCP1 were determined by ELISA. Results. Baseline distribution of Monl-2-3 was comparable between the 3 groups. Following acute exercise, \%Mon 2 and \%Mon3 increased significantly at the expense of a decrease in \%Mon1 in HS and in CKD. This response was significantly attenuated in CHF $(P<0.05)$. In HS only, MCP-1 levels increased following exercise; IL-6 levels were unchanged. Circulatory power was a strong and independent predictor of the changes in Mon1 $(\beta=-0.461, P<0.001)$ and Mon3 ( $\beta=0.449$, $P<0.001)$; and baseline LVEF of the change in Mon2 $(\beta=0.441, P<0.001)$. Conclusion. The response of monocytes to acute exercise is characterized by an increase in proangiogenic and proinflammatory Mon2 and Mon3 at the expense of phagocytic Monl. This exercise-induced monocyte subset response is mainly driven by hemodynamic changes and not by preexistent lowgrade inflammation.
\end{abstract}

\section{Introduction}

Monocytes are keystones of the immune system linking innate and adaptive immunity and are critical drivers in inflammatory diseases. Recently, 3 functionally distinct monocyte subsets were identified based on the expression of CD14 (lipopolysaccharide receptor), CD16 (FcyRIII receptor), and CCR2 (receptor for monocyte chemotactic protein-1 (MCP1)) $[1,2]$. Classical monocytes (Mon1, $\mathrm{CD} 14^{++} \mathrm{CD} 16^{-} \mathrm{CCR}^{+}$) are highly phagocytic and are important in first line defense. Intermediate monocytes (Mon2, $\mathrm{CD} 14^{++} \mathrm{CD}_{16}{ }^{+} \mathrm{CCR} 2^{+}$) possess proangiogenic properties, have been implicated in antigen processing and presentation, and produce high levels of pro- and anti-inflammatory mediators (TNF $\alpha$, IL-10). Nonclassical monocytes (Mon3, $\mathrm{CD} 14^{+} \mathrm{CD} 16^{++} \mathrm{CCR} 2^{-}$) have anti-inflammatory effects and play a role in the adaptive immune system as well as in patrolling the endothelial-blood interface $[1,3,4]$. 
Chronic low-grade inflammation, often objectified by an elevation in circulating levels of proinflammatory cytokines such as interleukin-6 (IL-6) and monocyte chemotactic protein-1 (MCP-1), has been recognized as the underlying factor in the development and progression of various diseases, including cardiovascular disease [5]. Chronic kidney disease (CKD) and chronic heart failure (CHF) share common mechanisms that explain the high cardiovascular morbidity and mortality, including inflammation and oxidative stressinduced vascular dysfunction $[6,7]$. Exercise training is a potent strategy for lowering long-term cardiovascular risk and morbidity in sedentary CKD patients [8] as well as mortality in CHF [9]. Randomized controlled exercise-intervention studies have shown that increased physical activity is associated with reduced systemic inflammation in the setting of CHF [10] and CKD [11]. It has been suggested that changes in the proportions of monocyte subsets contribute to this finding.

Although exercise training provides a strong anti-inflammatory effect, each intense exercise bout induces a transient increase in inflammatory markers, such as leukocytosis, monocytosis, and a raise in MCP-1 and IL-6 levels [12-14]. Nevertheless, endurance athletes have lower resting levels of inflammatory markers, including monocytes, in comparison to physically inactive adults, which suggests that the immune system adapts to repetitive exercise bouts [15].

In healthy subjects performing a short strenuous exercise bout, $\mathrm{CD}_{16}{ }^{+}$monocytes are mobilized from the marginal pool within 15 minutes [16]. The effect of acute exercise on the 3 monocytes subsets in CKD and CHF with demonstrated low-grade inflammation is currently unknown. The present study investigates whether monocyte subset distribution and their response to an acute exercise bout are influenced by the presence of chronic low-grade inflammation, the different homeostatic milieu of $\mathrm{CKD}$, or the hemodynamic alterations in CHF. We have the following aims: (1) to explore baseline differences in the monocyte subset distribution between $\mathrm{CKD}, \mathrm{CHF}$, and healthy controls; (2) to evaluate the effect of a single maximal exercise bout on the mobilization of Mon1, Mon2, and Mon3 in patients with CKD or CHF in comparison to healthy subjects; and (3) to investigate possible mediators of this response.

\section{Methods}

2.1. Subjects. Twenty sedentary CKD patients (estimated glomerular filtration rate $(\mathrm{eGFR})<60 \mathrm{~mL} / \mathrm{min} / 1.73 \mathrm{~m}^{2}$ or kidney damage for $\geq 3$ months, defined by structural or functional renal abnormalities), 20 sedentary CHF patients (left ventricular ejection fraction (LVEF) $\leq 45 \%$, NYHA classes II-III), and 15 healthy subjects (HS) (no relevant medical history, no pharmacological treatment, and normal ECG and echocardiographic assessment) were enrolled in this study. Patients were stable with regard to symptoms and therapy and were on standard medical treatment.

Exclusion criteria were active inflammatory or malignant disease and treatment with immunosuppressive agents. In the CKD group, the presence of cardiovascular disease, including coronary, peripheral, and cerebrovascular disease, served as an exclusion criterion. Likewise, in the $\mathrm{CHF}$ group, patients with impaired renal function $\left(\mathrm{eGFR}<60 \mathrm{~mL} / \mathrm{min} / 1.73 \mathrm{~m}^{2}\right)$ were excluded.

$\mathrm{CHF}$ was due to idiopathic dilated cardiomyopathy in $65 \%$ of patients; the remainder was caused by ischemic heart disease. Etiology of CKD included autosomal dominant polycystic kidney disease (25\%), the presence of a unique functional kidney (25\%), reflux nephropathy (20\%), IgA nephropathy $(10 \%)$, nephroangiosclerosis $(10 \%)$, focal segmental glomerulosclerosis (5\%), and obstructive nephropathy secondary to chronic lithiasis (5\%).

2.2. Study Design. Subjects were asked to refrain from excessive physical exertion for 24 hours prior to the study. They were called in for a symptom-limited cardiopulmonary exercise test (CPET) on a graded bicycle ergometer. Immediately before and 10 minutes after peak exercise, venous blood samples were drawn from an antecubital vein and collected in EDTA and serum separator tubes. Samples for flow cytometric analysis were processed within 1 hour after collection and serum was stored at $-80^{\circ} \mathrm{C}$ for batch analysis.

All subjects underwent standard transthoracic cardiac ultrasound for the assessment of left ventricular end-diastolic diameter (LVEDD), left ventricular ejection fraction (LVEF), diastolic function (E/é), and right atrial pressure (RAP) using an iE33 echocardiography scanner (Philips Medical Systems, Eindhoven, the Netherlands).

The study was conducted according to the principles outlined in the declaration of Helsinki and was approved by the Ethics Committee of the Antwerp University Hospital. All participants gave written informed consent.

2.3. Cardiopulmonary Exercise Testing. Exercise capacity was assessed by a symptom-limited graded exercise test on a bicycle ergometer (CKD and CHF patients: Cardiovit CS200 Ergo-Spiro, Schiller AG, Baar, Switzerland; healthy subjects: Excalibur Sport ergometer, Lode, Groningen, the Netherlands, and PowerCube-ergo, Ganshorn, Niederlauer, Germany). An individualized ramp protocol, starting with either 20 or 40 Watts and an incremental load of 10 or 20 Watts per minute, was chosen to ensure an optimal duration of the exercise test between 8 and 10 minutes. Twelve-lead ECG was recorded continuously and blood pressure was measured every 2 minutes. Breath-by-breath gas exchange measurements allowed online determination of ventilation (VE), oxygen uptake $\left(\mathrm{VO}_{2}\right)$, and carbon dioxide production $\left(\mathrm{VCO}_{2}\right)$. Peak oxygen consumption $\left(\mathrm{VO}_{2}\right.$ peak) was determined as the highest attained $\mathrm{VO}_{2}$ during the final 30 seconds of exercise. $\mathrm{VO}_{2}$ peak and maximal workload were also expressed as a percentage of the predicted value (\% predicted $\mathrm{VO}_{2}$ peak, $\%$ predicted Wattmax), according to the nomogram of Hansen et al. [17]. Subjects were encouraged to exercise upon exhaustion, according to the respiratory exchange ratio (RER) and identification of the anaerobic threshold (AT, V-slope method). Circulatory power $\left(\mathrm{VO}_{2}\right.$ peak $\times$ peak systolic blood pressure) and maximal work-economy (Wattmax $/ \mathrm{VO}_{2}$ peak) were calculated. 
2.4. Flow Cytometric Quantification of Monocyte Subsets. Monocyte subsets were defined as $\mathrm{CD}^{++}{ }^{++} \mathrm{CD} 16^{-}$ $\mathrm{CCR}^{+}$cells (Mon1), CD14 ${ }^{++} \mathrm{CD}^{+} 6^{+} \mathrm{CCR} 2^{+}$(Mon2), and $\mathrm{CD}_{1}{ }^{+} \mathrm{CD}_{16}{ }^{++} \mathrm{CCR} 2^{-}$(Mon3). Whole blood was incubated with fluorochrome-conjugated antibodies for 15 minutes in the dark. The following antibodies were used: anti-CD14 phycoerythrin (PE), anti-CD16 fluorescein isothiocyanate (FITC), anti-CCR2 allophycocyanin (APC) (R\&D Systems, Minnesota, USA), anti-CD45 allophycocyanin-Hilite 7 (APC-H7), and anti-CD86 peridinin chlorophyll proteincyanine 5.5 (PerCP-Cy5.5). All antibodies were purchased from $\mathrm{BD}$ Biosciences (Erembodegem, Belgium) unless stated otherwise. The optimal concentration for each antibody was determined prior to the study by titration assays.

After red blood cells lysis with BD lysing solution (BD Biosciences, Erembodegem, Belgium), the specimen was analysed on a BD FACSCantoII flow cytometer (BD Biosciences, Erembodegem, Belgium). Besides the regular forward scatter (FSC) threshold, an additional threshold was set on APC-H7 (below the CD45 APC-H7 fluorescence of granulocytes) to ensure proper recording of $\mathrm{CD}_{4} 5^{+}$events in this lyse-no-wash setting. A minimum of $200000 \mathrm{CD}^{2} 5^{+}$ events was recorded.

Analysis of monocyte subsets was done using BD FACSDiva software version 6.1 .2 by a single operator in analogy to the gating strategy of Shantsila et al. [3]. Primary gates were established for monocytes based on expression of the panmonocytic marker CD86 as well as scatter profile. Secondary gates were established within the monocyte gate to identify $\mathrm{CD} 14^{+}$and $\mathrm{CD} 16^{+}$cells. $\mathrm{CD}^{+} 6^{+}$cells were then separated in Mon 2 and Mon3 based on their CCR2 expression. Figure 1 shows a representative example of the applied gating strategy. Total leukocyte count was performed using an automated hematology analyzer (Advia 2120, Bayer HealthCare, Tarrytown, NY). Absolute count of monocytes and their subsets was calculated by multiplying the respective percentages acquired by flow cytometry by the total leukocyte count. Monocyte subsets were expressed as cells $/ \mu \mathrm{L}$ and as a percentage of total monocyte count. For lymphocyte and neutrophil percentage, results of the automated hematology analyzer were used.

2.5. Biochemical Assays. Creatinine, total cholesterol, highdensity lipoprotein (HDL), low-density lipoprotein (LDL), and triglycerides were measured using routine laboratory techniques. Estimated glomerular filtration rate (eGFR) was calculated using the CKD-EPI formula [18].

Serum levels of MCP-1 and IL- 6 were analysed in batch by sandwich enzyme-linked immunosorbent assay (Quantikine ELISA kit, R\&D Systems, Minnesota, USA). For MCP-1, the intra-assay coefficient of variation $(\mathrm{CV})$ was $<10 \%$ with a sensitivity of $1.7 \mathrm{pg} / \mathrm{mL}$. For IL-6, the intra-assay CV was $<20 \%$ with a sensitivity of $0.7 \mathrm{pg} / \mathrm{mL}$.

2.6. Statistical Analysis. Continuous data are expressed as mean \pm standard deviation (SD). Normality of data was assessed using a one-sample Kolmogorov Smirnov. Baseline characteristics were compared using Chi-square test or one-way ANOVA followed by the Sidak post hoc test for multiple comparison correction.

Different trends over time between groups (interaction) were assessed by two-way repeated measures ANOVA. Differences over time within each group were assessed by paired samples $t$-tests. One-way ANOVA of the percentual changes in the monocyte subsets was used to determine the magnitude of the exercise-induced effects and was followed by the Sidak post hoc test. Pearson correlation coefficients were used where appropriate. Multiple linear regression analysis was applied to investigate the independent association between exercise parameters and monocyte numbers. All analyses were performed using SPSS version 22 (SPSS Inc., Chicago, IL, USA) and a $P$ value of $<0.05$ was considered statistically significant.

\section{Results and Discussion}

\subsection{Results}

3.1.1. Baseline Characteristics of Subjects. Table 1 summarizes the baseline characteristics of the 3 groups. Age, sex, and BMI were comparable between groups. CKD patients presented with a mean eGFR $44.4 \pm 19.7 \mathrm{~mL} / \mathrm{min} / 1.73 \mathrm{~m}^{2}$. CHF patients, with a mean LVEF of $31.1 \pm 10.6 \%$, were characterized by elevated atrial and left ventricular filling pressures.

All subjects performed a maximal exercise test, as was objectified by a RER value $>1.15$. Aerobic exercise capacity ( $\mathrm{VO}_{2}$ peak) and maximal workload were significantly lower in $\mathrm{CKD}$ and CHF patients compared to HS. Compared to CKD and HS, patients with $\mathrm{CHF}$ had a reduced hemodynamic response with a lower peak heart rate, systolic blood pressure, and circulatory power (Table 1).

3.1.2. Distribution of Monocyte Subsets and Levels of Inflammatory Proteins at Baseline. Total leukocyte count was within normal range in the 3 groups $\left(4.3-10.010^{6} / \mathrm{mL}\right)$, but $\mathrm{CHF}$ patients had significantly higher white blood cell counts compared to CKD patients (Table 2). Whereas the distribution of neutrophils and lymphocytes was comparable between groups, CHF patients had a higher percentage of total monocytes and consequently a higher absolute count of all monocyte subsets. Distribution of the monocyte subsets was comparable between the groups: Monl comprised the largest percentage, followed by Mon3 and then Mon2 (Table 2).

Levels of MCP-1 were significantly higher in CKD and CHF compared to HS. IL-6 levels did not differ significantly between groups but were related to MCP-1 levels ( $r=0.407$, $P<0.001)$ and $\%$ Mon2 $(r=0.312, P=0.031)$. Neither total leukocyte nor monocyte count was correlated to levels of IL6 or MCP-1. Considering all groups, baseline levels of MCP-1 and IL-6 were significantly related to $\mathrm{VO}_{2}$ peak (MCP-1 $r=$ $-0.330, P=0.017$; IL-6 $r=-0.364, P=0.013$, resp.). No relation was found between leukocyte and monocyte counts or monocyte distribution with $\mathrm{VO}_{2}$ peak.

3.1.3. Effect of an Acute Exercise Bout on Leukocyte and Monocyte Distribution. Table 3 demonstrates that absolute monocyte count increased significantly and comparably in 

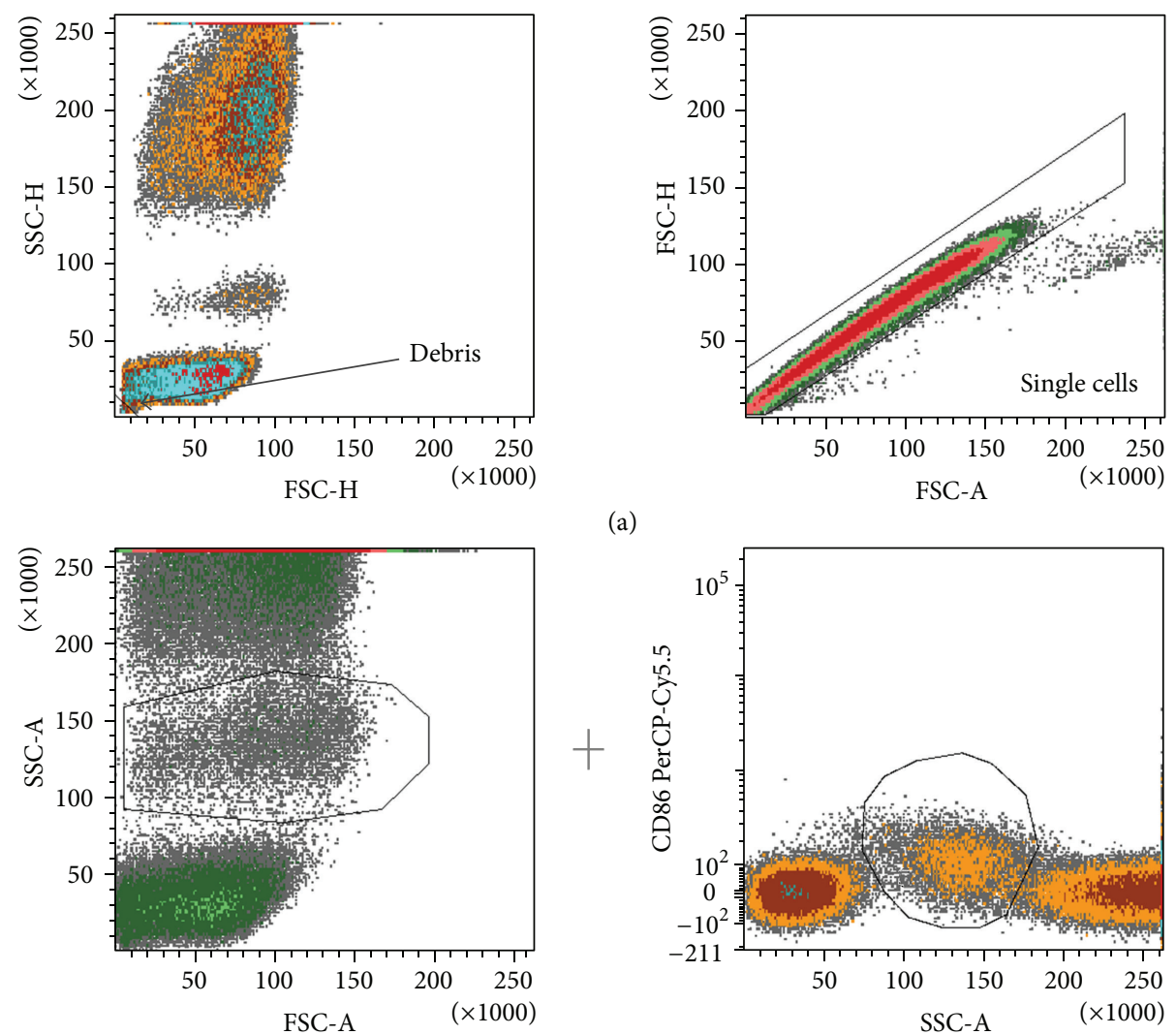

(a)
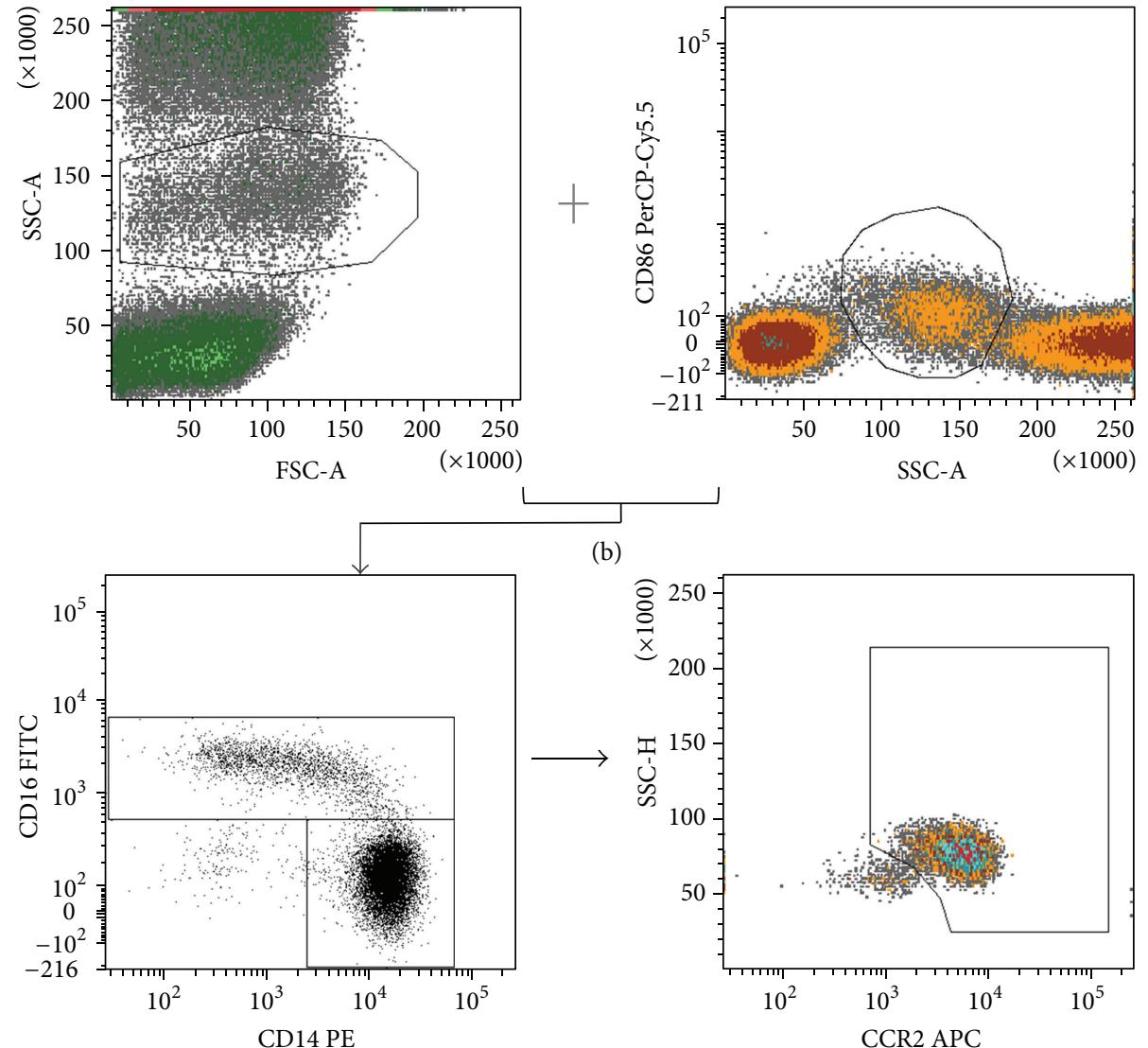

(b)

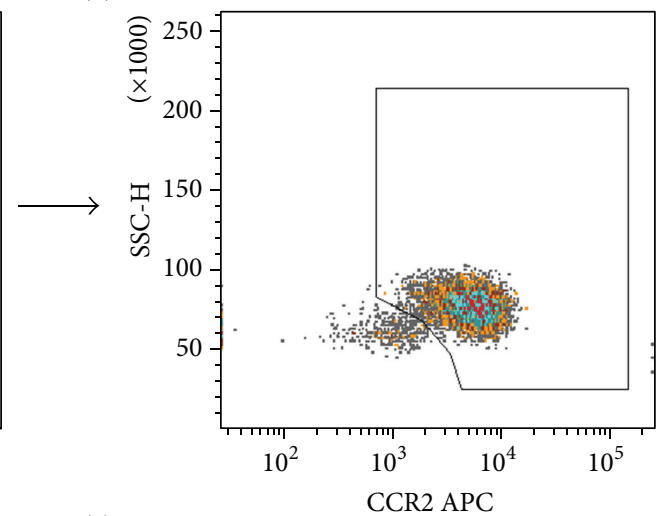

(c)
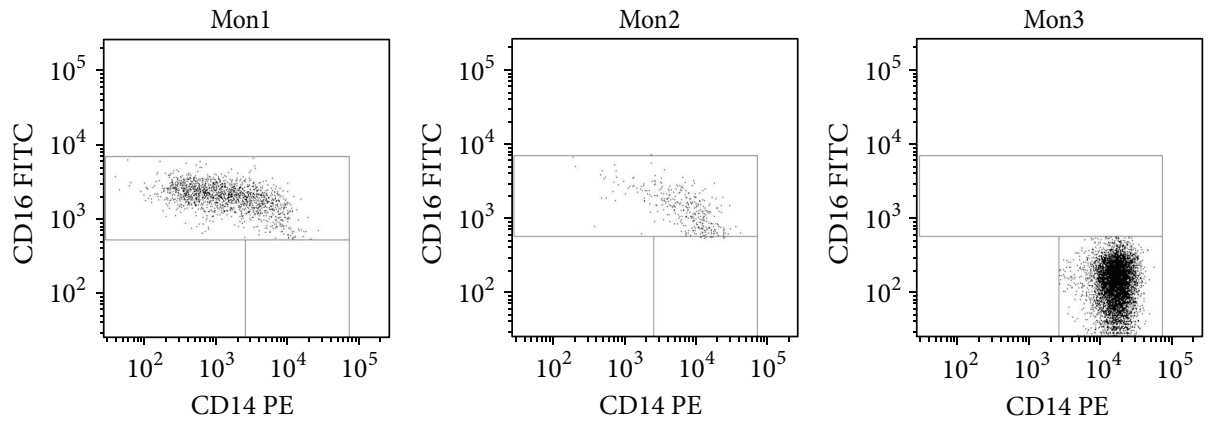

(d)

FIGURE 1: Gating strategy. Gating strategy and presentation of monocyte subsets. (a) Exclusion of debris and doublets. (b) Identification of monocytes based on the forward scatter (FSC) versus side scatter (SSC) plot and CD86 positivity. (c) Separation of monocytes in CD16 positive and CD16 negative monocytes and subsequent distinction between Mon2 and Mon3, based on CCR2 expression. The plot shows all monocyte subsets, with Mon1 as per definition in the CCR2 ${ }^{+}$gate. (d) Respective location of the monocyte subsets on CD14 versus CD16 plot. 
TABLE 1: Baseline characteristics of subjects.

\begin{tabular}{|c|c|c|c|c|}
\hline & HS $(n=15)$ & $\mathrm{CKD}(n=20)$ & $\mathrm{CHF}(n=20)$ & $P$ value \\
\hline Age (years) & $43.5 \pm 5.0$ & $51.3 \pm 15.6$ & $51.2 \pm 9.3$ & 0.08 \\
\hline Gender (F/M) & $6 / 9$ & $12 / 8$ & $7 / 13$ & 0.25 \\
\hline Body mass index $\left(\mathrm{kg} / \mathrm{m}^{2}\right)$ & $24.2 \pm 2.3$ & $26.1 \pm 5.1$ & $26.6 \pm 3.8$ & 0.18 \\
\hline Systolic BP (mmHg) & $123 \pm 13$ & $122+13$ & $102 \pm 18^{* \dagger}$ & $<0.001$ \\
\hline Diastolic BP (mmHg) & $77 \pm 9$ & $77 \pm 9$ & $69 \pm 10^{* \dagger}$ & $<0.05$ \\
\hline \multicolumn{5}{|l|}{ Biochemistry } \\
\hline $\mathrm{eGFR}\left(\mathrm{mL} / \mathrm{min} / 1.73 \mathrm{~m}^{2}\right)$ & $99.0 \pm 11.3$ & $44.4 \pm 19.7^{*}$ & $88.7 \pm 12.9^{\dagger}$ & $<0.001$ \\
\hline Total cholesterol (mg/dL) & $172.7 \pm 22.2$ & $173.8 \pm 24.1$ & $178.3 \pm 8.9$ & 0.83 \\
\hline $\mathrm{HDL}(\mathrm{mg} / \mathrm{dL})$ & $58.9 \pm 13.6$ & $57.7 \pm 18.8$ & $46.8 \pm 11.3^{* \dagger}$ & $<0.05$ \\
\hline $\mathrm{LDL}(\mathrm{mg} / \mathrm{dL})$ & $111.4 \pm 34.8$ & $98.4 \pm 20.7$ & $99.3 \pm 24.7$ & 0.32 \\
\hline \multicolumn{5}{|l|}{ Echocardiography } \\
\hline LVEF (\%) & $65 \pm 0$ & $62.9 \pm 8.2$ & $31.1 \pm 10.6^{* \dagger}$ & $<0.001$ \\
\hline $\operatorname{LVEDD}(\mathrm{mm})$ & $49.8 \pm 4.4$ & $47.8 \pm 4.9$ & $59.9 \pm 13.2^{* \dagger}$ & $<0.001$ \\
\hline E/é & $9.2 \pm 1.3$ & $9.7 \pm 2.4$ & $17.4 \pm 8.9^{* \dagger}$ & $<0.001$ \\
\hline RAP (mmHg) & $5.4 \pm 1.4$ & $4.6 \pm 1.1$ & $6.5 \pm 2.9^{\dagger}$ & $<0.001$ \\
\hline \multicolumn{5}{|l|}{ Medication use } \\
\hline Beta-blockers (\%) & l & 35 & 100 & $<0.001$ \\
\hline Diuretics (\%) & l & 15 & 70 & $<0.05$ \\
\hline Acetylsalicylic acid (\%) & I & 5 & 40 & $<0.05$ \\
\hline Statins (\%) & I & 55 & 50 & 0.5 \\
\hline ACE-inhibitors/ARB (\%) & l & 60 & 95 & $<0.05$ \\
\hline \multicolumn{5}{|l|}{ CPET-derived parameters } \\
\hline RER & $1.24 \pm 0.11$ & $1.38 \pm 0.13^{*}$ & $1.34 \pm 0.12$ & $<0.05$ \\
\hline $\mathrm{VO}_{2}$ peak $(\mathrm{mL} / \mathrm{kg} / \mathrm{min})$ & $37.40 \pm 9.38$ & $25.54 \pm 7.54^{*}$ & $22.19 \pm 5.96^{*}$ & $<0.001$ \\
\hline$\%$ predicted $\mathrm{VO}_{2}$ peak $(\%)$ & $107 \pm 22$ & $84 \pm 20^{*}$ & $71 \pm 13^{*}$ & $<0.001$ \\
\hline Maximal workload (Watt) & $246 \pm 85$ & $152 \pm 50^{*}$ & $132 \pm 49^{*}$ & $<0.001$ \\
\hline$\%$ predicted Wattmax (\%) & $123 \pm 26$ & $95 \pm 29^{*}$ & $81 \pm 19^{*}$ & $<0.001$ \\
\hline $\mathrm{VO}_{2}$ at $\mathrm{AT}(\mathrm{mL} / \mathrm{kg} / \mathrm{min})$ & $31.42 \pm 8.14$ & $24.96 \pm 6.75$ & $20.08 \pm 6.36^{*}$ & 0.001 \\
\hline Peak heart rate (bpm) & $170 \pm 14$ & $154 \pm 27$ & $135 \pm 21^{* \dagger}$ & $<0.001$ \\
\hline Peak systolic BP (mmHg) & $189 \pm 35$ & $188 \pm 21$ & $137 \pm 27^{* \dagger}$ & $<0.001$ \\
\hline Peak diastolic BP (mmHg) & $80 \pm 11$ & $82 \pm 16$ & $73 \pm 14$ & 0.156 \\
\hline Work economy (Watt/(mL/kg/min)) & $6.8 \pm 1.5$ & $6.1 \pm 1.3$ & $5.9 \pm 1.3$ & 0.19 \\
\hline Circulatory power $\left(\mathrm{mmHg} \cdot \mathrm{mLVO}_{2} /(\mathrm{kg} / \mathrm{min})\right)$ & $7017 \pm 2504$ & $4828 \pm 1669^{*}$ & $3097 \pm 1191^{* \dagger}$ & $<0.001$ \\
\hline$T_{1 / 2} \mathrm{VO}_{2}$ peak (seconds) & $120 \pm 45$ & $176 \pm 43^{*}$ & $199 \pm 32^{*}$ & $<0.001$ \\
\hline Exercise duration (sec) & $749 \pm 230$ & $424 \pm 130^{*}$ & $447 \pm 120^{*}$ & $<0.001$ \\
\hline
\end{tabular}

Data are mean \pm SD. $P$ value for comparison of groups (ANOVA). ${ }^{*}$ Different from HS, $P<0.05 .{ }^{\dagger}$ Different from CKD, $P<0.05$.

BP: blood pressure; eGFR: estimated glomerular filtration ratio; HDL: high-density lipoprotein; LDL: low-density lipoprotein; LVEF: left ventricular ejection fraction; LVEDD: left ventricular end-diastolic diameter; RAP: right atrial pressure; RER: respiratory exchange ratio; $\mathrm{VO}_{2}$ peak: peak oxygen uptake; $\mathrm{VO}_{2}$ at AT: oxygen uptake at anaerobic threshold; $T_{1 / 2} \mathrm{VO}_{2}$ peak: $\mathrm{VO}_{2}$ peak half-time; ACE: angiotensin converting enzyme; ARB: angiotensin receptor blocker.

HS, CKD, and CHF patients 10 minutes following peak exercise. This was due to a general exercise-induced leukocytosis. Only in CHF, a significant increase in percentage of monocytes contributed to this leukocytosis.

In all 3 groups, percentage of Mon1 decreased, whereas Mon2 and Mon3 increased after a single bout (with exception of Mon2 in CHF, Table 3). Figure 2 illustrates that the magnitude of this exercise-induced effect on monocyte subsets is different in the 3 groups. The overall exercise-induced response on monocyte subsets was comparable between HS and CKD but was blunted in patients with $\mathrm{CHF}$ ( $P$ for interaction $<0.05$ for all subsets). In CHF, the decrease in Mon1 was less prominent, the increase in Mon2 was nearly absent, and the increase in Mon3 again was less pronounced.

3.1.4. Effect of an Acute Exercise Bout on Levels of MCP-1 and $I L-6$. Whereas an acute exercise bout did not alter levels of MCP-1 in patients with CKD and CHF, MCP-1 was significantly higher in HS following exercise $(P=0.004$ for interaction, Figure 2(e)). Postexercise levels in MCP-1 were comparable between groups $(P>0.05)$. Increases in levels of IL-6 were observed in all groups but failed to reach the level of significance (HS $P=0.08$; CKD $P=0.644$; CHF $P=0.063$, Figure 2(f)). 
TABLE 2: Distribution of monocyte subsets and levels of inflammatory proteins at baseline.

\begin{tabular}{|c|c|c|c|c|}
\hline & HS $(n=15)$ & $\mathrm{CKD}(n=20)$ & $\mathrm{CHF}(n=20)$ & $P$ value \\
\hline \multicolumn{5}{|l|}{ Leukocytes } \\
\hline WBC count $(10 E 6 / \mathrm{mL})$ & $7.17 \pm 1.60$ & $5.76 \pm 1.45$ & $8.24 \pm 1.82^{\dagger}$ & $<0.001$ \\
\hline \multicolumn{5}{|l|}{ WBC formula (\% of leukocytes) } \\
\hline \%neutrophils & $65.1 \pm 10.6$ & $63.1 \pm 7.8$ & $59.8 \pm 9.7$ & 0.24 \\
\hline \%lymphocytes & $24.8 \pm 8.7$ & $24.2 \pm 6.8$ & $27.5 \pm 8.3$ & 0.38 \\
\hline \%monocytes & $6.28 \pm 1.24$ & $6.16 \pm 1.35$ & $7.72 \pm 1.88^{* \dagger}$ & $<0.05$ \\
\hline \multicolumn{5}{|l|}{ Monocytes } \\
\hline Monocyte count (cells/ $\mu \mathrm{L}$ ) & $450 \pm 128$ & $352 \pm 103$ & $628 \pm 159^{* \dagger}$ & $<0.001$ \\
\hline \multicolumn{5}{|c|}{ Monocyte subsets (\% of monocytes) } \\
\hline \%Monl & $88.09 \pm 4.73$ & $88.48 \pm 4.27$ & $87.34 \pm 3.54$ & 0.67 \\
\hline \%Mon2 & $4.51 \pm 2.05$ & $3.56 \pm 1.69$ & $4.74 \pm 2.46$ & 0.18 \\
\hline \%Mon3 & $7.39 \pm 3.17$ & $7.95 \pm 3.61$ & $7.92 \pm 2.19$ & 0.83 \\
\hline \multicolumn{5}{|l|}{ Monocyte subsets (cells/L) } \\
\hline Mon1 & $395.2 \pm 107$ & $311.6 \pm 93.8$ & $550.3 \pm 143.9^{* \dagger}$ & $<0.001$ \\
\hline Mon2 & $20.7 \pm 13.5$ & $13.1 \pm 8.7$ & $29.3 \pm 17.1^{\dagger}$ & $<0.01$ \\
\hline Mon3 & $34.1 \pm 20.9$ & $27.3 \pm 13.9$ & $49.3 \pm 17.3^{* \dagger}$ & $<0.01$ \\
\hline \multicolumn{5}{|l|}{ Inflammatory cytokines } \\
\hline MCP-1 (pg/mL) & $330 \pm 163$ & $446 \pm 95^{*}$ & $420 \pm 119^{*}$ & 0.028 \\
\hline IL-6 (pg/mL) & $1.17 \pm 1.72$ & $1.63 \pm 1.43$ & $1.41 \pm 1.33$ & 0.69 \\
\hline
\end{tabular}

Data are mean \pm SD. $P$ value for comparison of groups (ANOVA). ${ }^{*}$ Different from HS, $P<0.05$. ${ }^{\dagger}$ Different from CKD, $P<0.05$.

MCP-1: monocyte chemoattractant protein-1; IL-6: interleukin-6.

TABLE 3: Effect of an acute exercise bout on leukocyte and monocyte distribution.

\begin{tabular}{|c|c|c|c|c|c|}
\hline & Pre & Post & $\%$ change & $P$ value for pre-postcomparison & $P$ value for interaction \\
\hline \multicolumn{6}{|c|}{ Leukocytes $(10 E 6 / \mathrm{mL})$} \\
\hline HS & $7.17 \pm 1.60$ & $11.79 \pm 1.99$ & $67.28 \pm 26.54$ & $<0.001$ & \multirow{3}{*}{$<0.001$} \\
\hline CKD & $5.76 \pm 1.45$ & $9.95 \pm 2.39$ & $76.65 \pm 37.1$ & 0.006 & \\
\hline $\mathrm{CHF}$ & $8.24 \pm 1.82$ & $10.21 \pm 1.66$ & $26.47 \pm 18.0^{* \dagger}$ & $<0.001$ & \\
\hline \multicolumn{6}{|c|}{ Monocytes (cells/ $\mu L)$} \\
\hline HS & $450 \pm 128$ & $728 \pm 206$ & $62.79 \pm 21.36$ & $<0.001$ & \multirow{3}{*}{0.26} \\
\hline CKD & $352 \pm 103$ & $598 \pm 162$ & $75.95 \pm 44.68$ & $<0.001$ & \\
\hline $\mathrm{CHF}$ & $628 \pm 159$ & $833 \pm 218$ & $34.85 \pm 23.89$ & $<0.001$ & \\
\hline \multicolumn{6}{|c|}{ Monocytes (\% of leukocytes) } \\
\hline HS & $6.28 \pm 1.24$ & $6.13 \pm 1.16$ & $-1.92 \pm 9.04$ & NS & \multirow{3}{*}{$<0.001$} \\
\hline CKD & $6.16 \pm 1.35$ & $6.17 \pm 1.93$ & $0.09 \pm 17.89$ & NS & \\
\hline $\mathrm{CHF}$ & $7.72 \pm 1.88$ & $8.21 \pm 2.15$ & $6.55 \pm 9.74$ & 0.024 & \\
\hline \multicolumn{6}{|c|}{ Mon1 (\% of total monocytes) } \\
\hline HS & $88.09 \pm 4.73$ & $83.12 \pm 5.98$ & $-5.7 \pm 3.3$ & $<0.001$ & \multirow{3}{*}{0.001} \\
\hline CKD & $88.48 \pm 4.27$ & $85.27 \pm 3.73$ & $-3.5 \pm 3.1$ & $<0.001$ & \\
\hline $\mathrm{CHF}$ & $87.34 \pm 3.54$ & $85.86 \pm 4.25$ & $-1.7 \pm 2.4^{*}$ & 0.004 & \\
\hline \multicolumn{6}{|c|}{ Mon2 (\% of total monocytes) } \\
\hline HS & $4.51 \pm 2.05$ & $5.62 \pm 2.30$ & $28.9 \pm 31.4$ & $<0.001$ & \multirow{3}{*}{0.002} \\
\hline CKD & $3.56 \pm 1.69$ & $4.43 \pm 1.62$ & $38.3 \pm 48.1$ & 0.001 & \\
\hline $\mathrm{CHF}$ & $4.74 \pm 2.46$ & $4.77 \pm 2.22$ & $5 \pm 17.2^{\dagger}$ & NS & \\
\hline \multicolumn{6}{|c|}{ Mon3 (\% of total monocytes) } \\
\hline HS & $7.39 \pm 3.17$ & $11.26 \pm 4.59$ & $57.2 \pm 31.3$ & $<0.001$ & \multirow{3}{*}{0.004} \\
\hline CKD & $7.95 \pm 3.61$ & $10.29 \pm 3.0$ & $43.2 \pm 45.3$ & $<0.001$ & \\
\hline $\mathrm{CHF}$ & $7.92 \pm 2.19$ & $9.37 \pm 3.13$ & $17.2 \pm 17.2^{*}$ & $<0.001$ & \\
\hline
\end{tabular}

Data are mean \pm SD. $P$ value for within-group (paired samples $t$-test) and between-group comparison (repeated measures ANOVA). ${ }^{*}$ Different from HS, $P<$ 0.05. ${ }^{\dagger}$ Different from CKD, $P<0.05$. 


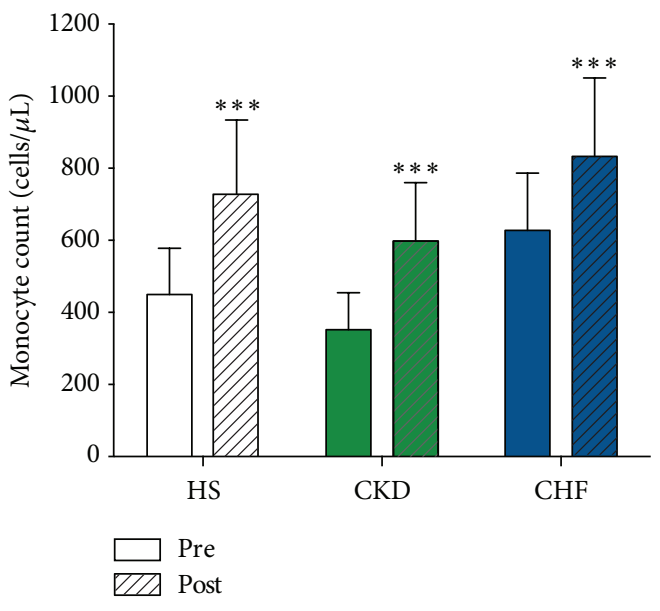

(a)

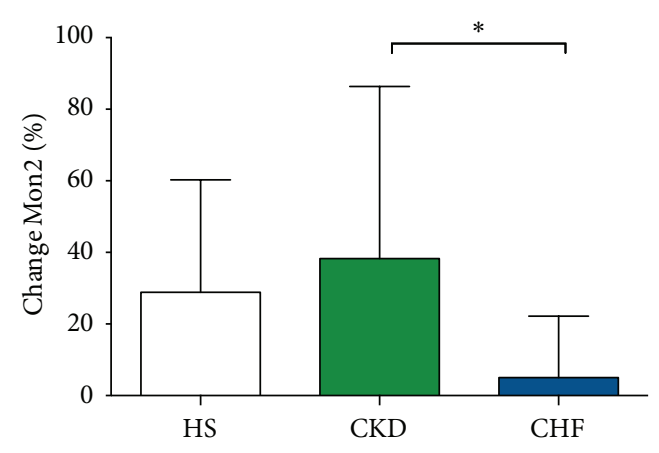

(c)

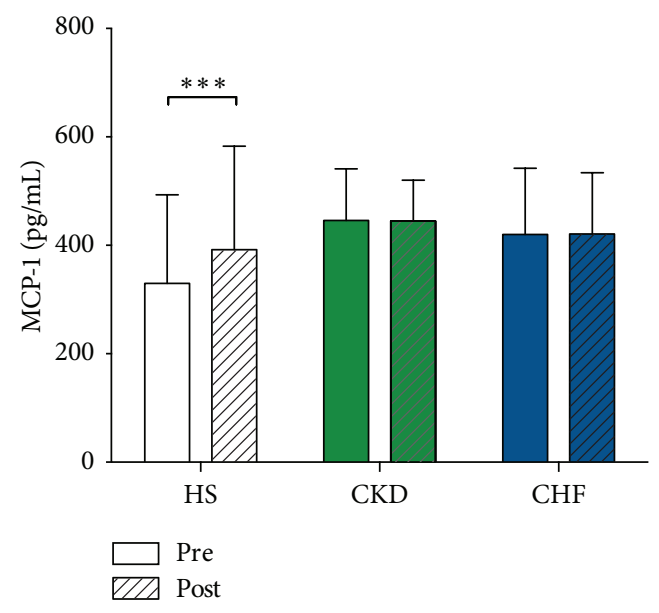

(e)

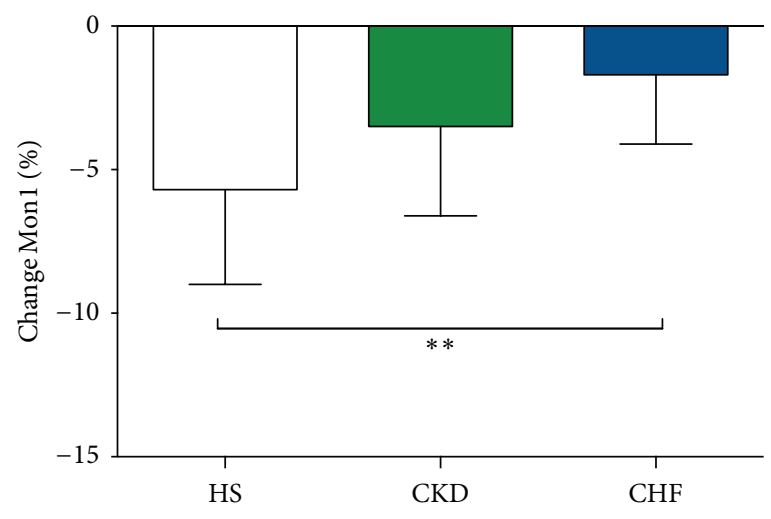

(b)

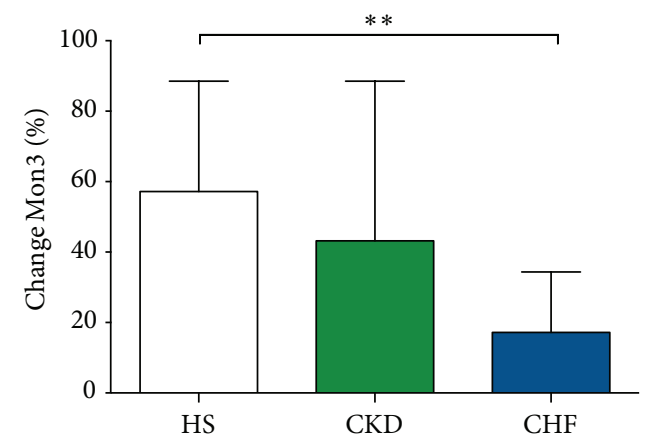

(d)

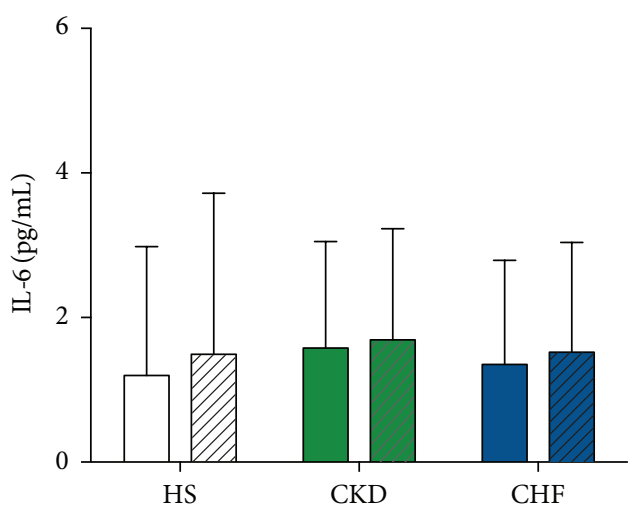

$\square$ Pre

(f)

Figure 2: Magnitude of the exercise-induced effect on monocyte count, monocyte subsets, and serum MCP-1 and IL-6 levels. (a) Following peak exercise, absolute monocyte count increased significantly in all groups. (b-d) Within the total monocyte count, the percentage of Monl decreased in all three groups with a parallel increase in Mon2 and Mon3 (with exception of Mon2 in CHF). Between-group analysis revealed that the overall response of the monocyte subsets was comparable between HS and CKD but was significantly blunted for patients with CHF ( $P$ for interaction $<0.05$ for all subsets). (e) Following peak exercise, MCP-1 levels increased significantly in HS but remained unchanged in patients with CKD and CHF ( $P=0.004$ for interaction). (f) Increase in IL-6 levels were observed in all groups but failed to reach the level of significance (HS $P=0.08$; CKD $P=0.644$; CHF $P=0.063$ ). Changes in monocyte subset are expressed as $\%$ change from baseline. ${ }^{* * *} P<0.001,{ }^{* *} P<0.01$, and ${ }^{*} P<0.05$. 
TABLE 4: Relation of changes in monocyte subsets with baseline, exercise, and inflammation-related parameters.

\begin{tabular}{|c|c|c|c|c|c|c|c|c|c|c|}
\hline & \multicolumn{2}{|c|}{ Change WBC count } & \multicolumn{2}{|c|}{ Change Mon count } & \multicolumn{2}{|c|}{ Change \%Mon1 } & \multicolumn{2}{|c|}{ Change \%Mon 2} & \multicolumn{2}{|c|}{ Change \%Mon3 } \\
\hline & $r$ & $P$ & $r$ & $P$ & $r$ & $P$ & $r$ & $P$ & $r$ & $P$ \\
\hline \multicolumn{11}{|l|}{ Baseline parameters } \\
\hline Systolic BP & 0.438 & $<0.001$ & 0.366 & 0.007 & -0.215 & 0.122 & 0.213 & 0.125 & 0.364 & 0.007 \\
\hline Diastolic dysfunction (E/é) & -0.403 & 0.003 & -0.333 & 0.015 & 0.367 & 0.007 & -0.327 & 0.017 & -0.359 & 0.008 \\
\hline Systolic function (LVEF) & 0.579 & $<0.001$ & 0.429 & $<0.001$ & -0.422 & 0.002 & 0.430 & $<0.001$ & 0.378 & 0.005 \\
\hline eGFR & -0.28 & 0.038 & -0.245 & 0.072 & -0.111 & 0.420 & -0.177 & 0.195 & 0.075 & 0.588 \\
\hline MCP-1 & -0.077 & 0.582 & -0.066 & 0.633 & 0.075 & 0.589 & 0.161 & 0.245 & -0.050 & 0.722 \\
\hline \multicolumn{11}{|c|}{ Exercise-related hemodynamic parameters } \\
\hline $\mathrm{VO}_{2}$ peak & 0.418 & 0.002 & 0.308 & 0.025 & -0.536 & $<0.001$ & 0.218 & 0.117 & 0.504 & $<0.001$ \\
\hline Circulatory power & 0.477 & $<0.001$ & 0.309 & 0.031 & -0.466 & $<0.001$ & 0.229 & 0.114 & 0.451 & $<0.001$ \\
\hline Peak HR & 0.588 & $<0.001$ & 0.418 & 0.002 & -0.409 & 0.002 & 0.223 & 0.101 & 0.311 & 0.021 \\
\hline Peak systolic BP & 0.523 & $<0.001$ & 0.323 & 0.021 & -0.389 & 0.005 & 0.291 & 0.038 & 0.352 & 0.011 \\
\hline \multicolumn{11}{|l|}{ Inflammation-related parameters } \\
\hline IL-6 change & 0.323 & 0.037 & 0.046 & 0.774 & -0.093 & 0.557 & 0.015 & 0.923 & -0.009 & 0.954 \\
\hline MCP-1 change & 0.205 & 0.140 & 0.190 & 0.172 & -0.391 & 0.004 & -0.027 & 0.846 & 0.319 & 0.020 \\
\hline
\end{tabular}

BP: blood pressure; LVEF: left ventricular ejection fraction; $\mathrm{VO}_{2}$ peak: peak oxygen uptake; HR: heart rate; IL-6: interleukin-6; MCP-1: monocyte chemoattractant protein-1.

$r$ : Pearson correlation coefficient. Significant correlations are presented in bold.

3.1.5. Relation of Changes in Leukocytes and Monocyte Subsets with Baseline, Exercise, and Inflammation-Related Parameters. Considering all groups, baseline hemodynamic parameters such as systolic blood pressure, diastolic and systolic function, were strongly associated with the change of leukocyte and monocyte count and monocyte subset distribution after an acute exercise bout (Table 4). Subjects with an impaired diastolic function (higher E/é) and an impaired systolic function (lower LVEF) showed overall a less prominent change in leukocytes, monocytes, and monocyte subsets (decrease in \%Mon1, increase in \%Mon2 and \%Mon3). Renal function (eGFR) or inflammatory status (MCP-1) did not relate to the magnitude of this effect.

Exercise-related hemodynamic parameters, such as $\mathrm{VO}_{2}$ peak, circulatory power, and peak systolic blood pressure, were all related to changes in leukocyte or monocyte (subset) distribution (Table 4). To investigate the independent association between exercise-related parameters and monocyte subset change, a multiple linear regression analysis was performed, adjusting for age, sex, the use of beta-blockade, baseline systolic blood pressure, and diastolic and systolic function. $\mathrm{VO}_{2}$ peak remained a strong predictor of the change in Mon1 $(\beta=-0.495, P<0.001)$ and $\operatorname{Mon} 3(\beta=$ $0.468, P=0.001)$. In line, circulatory power remained negatively associated with the change in Mon1 $(\beta=-0.461, P<$ $0.001)$ and positively with the change in Mon3 $(\beta=0.449$, $P<0.001)$. For the change in Mon2, baseline LVEF emerged as the strongest response predictor $(\beta=0.441, P<0.001)$. The same was true when correcting (in separate models) for the use of statins, ACE-inhibitors/ARB, diuretics, and acetylsalicylic acid.

A significant relation between the exercise-induced change in MCP-1 and Monl and Mon3 was observed (Table 4).
However, after correction for $\mathrm{VO}_{2}$ peak, which was significantly correlated to change in MCP-1 $(r=0.511, P<0.001)$, this relation was lost.

3.2. Discussion. The present study investigates whether monocyte subset distribution and their response to an acute exercise bout are influenced by the presence of chronic low-grade inflammation (CKD and $\mathrm{CHF}$ ), the presence of CKD per se (specific internal milieu), or CHF (specific hemodynamic alterations).

Several findings emerge from this study.

(i) The relative distribution of the monocyte subsets (Mon 1-2-3) is comparable in healthy subjects, CKD patients, and $\mathrm{CHF}$ patients. However, in $\mathrm{CHF}$, the absolute monocyte count is significantly higher.

(ii) Following a single bout of maximal exercise, the percentage of Mon 2 and Mon3 increases at the expense of a decrease in Mon1. This response is clearly blunted in patients with $\mathrm{CHF}$ despite the fact that they performed a maximal exercise test and that they reached a similar $\mathrm{VO}_{2}$ peak and maximal workload compared to the CKD patients.

(iii) $\mathrm{VO}_{2}$ peak and circulatory power emerge as strong predictors of the changes in Mon1 and Mon3, independent of beta-blocker use.

3.2.1. Heterogeneity of Monocyte Subsets in Two Models of Chronic Disease with Low-Grade Inflammation. Before the nomenclature consensus in 2010, $\mathrm{CD}^{+} 6^{+}$monocytes (Mon2 and Mon3) were frequently studied collectively as proinflammatory monocytes, based on their cytokine expression profile 
and higher potency in antigen presentation. In addition to the fact that $\mathrm{CD}^{+} 6^{+}$monocyte count is increased in several inflammatory conditions, $\mathrm{CD}^{+} 6^{+}$monocytes have been clinically and mechanistically implicated in the pathophysiology of human cardiovascular disease [19].

The recognition of intermediate monocytes (Mon2) as a distinct subset urged for further unravelling of the function and behaviour of the different monocyte subtypes. Mon2 phenotypically resemble the previously reported proangiogenic monocytes, with the expression of receptors to proangiogenic factors (Tie2, CXCR4, and VEGFR1/2) [3, 20]. Indeed, bone marrow-derived $\mathrm{CD} 14^{+} \mathrm{Tie}^{+} \mathrm{CD} 34^{-}$cells are able to adhere on injured endothelium in a MCP-1-dependent manner, leading to reendothelialisation [21]. In contrast to their proangiogenic features, Mon2 possess a higher proinflammatory capacity compared to Mon3 [1] and selectively express CCR5, a marker that has been implicated in atherosclerosis [22]. These two characteristics could add to their possible unfavorable effect in cardiovascular disease. Indeed, in patients with CKD, in whom cardiovascular risk is known to be very high, as well as in patients at risk for coronary artery disease, elevated Mon2 counts are independent predictors of future cardiac events $[23,24]$. In contrast, in the context of acute heart failure, lower Mon 2 counts are independent predictors of increased mortality and repeat hospitalization [25]. These apparently conflicting data suggest diverse roles of the Mon2 subsets in different underlying disease processes.

In the present study, we show that the distribution of the 3 monocyte subsets (Mon1 > Mon3 > Mon2) is maintained in the presence of CKD or CHF. For CHF, this confirms the findings of Wrigley et al. [25], but it is in contrast with previous reports on a higher percentage of $\mathrm{CD} 14^{++} \mathrm{CD} 16^{+}$monocytes [26] or the CD14 ${ }^{\text {dim }} \mathrm{CD} 16^{+}$subset [27] in patients with $\mathrm{CHF}$ in comparison to healthy subjects. These discrepant findings possibly could be explained by the use of a different immunophenotypical definition between studies or by differences in the studied populations (older patients and a predominance of ischemic cardiomyopathy in the study by Barisione et al.). In analogy to the study of Wrigley et al., we used a multiparametric flow cytometric technique with an iterative gating strategy for enumeration of the different subsets, whereas the other studies used a straightforward twocolour panel approach. As illustrated by Zawada et al. [28], a pan-monocytic marker (CD86) is required to correctly identify the monocytes and to distinguish the $\mathrm{CD}^{+} 6^{+}$monocytes from other CD16 expressing leukocytes, such as neutrophils and natural killer cells. Second, we applied a colouring and gating strategy based on the differential expression of CCR2 for correct distinction between Mon2 and Mon3, adapted from the publication by Shantsila et al. [3].

In patients with CHF total monocyte count was increased, resulting in a parallel increase of all 3 subsets counts, again confirming the data of Wrigley et al. [25]. Limited data in $\mathrm{CHF}$ suggest that an increase in total monocyte number predicts worse outcome [29]. Such an association is far less explored in CKD and healthy subjects [30]. It is plausible that, in our study, we underestimated monocyte count in CKD patients, since we preselected CKD patients with the best cardiovascular prognosis by excluding any cardiovascular history. Therefore, total monocyte counts were rather low and no increase in Mon2 count was detected in CKD.

\subsubsection{The Effect of an Acute Exercise Bout on Monocyte} Subsets is Attenuated in CHF. It has been shown previously in healthy subjects that monocyte subsets behave differently in response to a physical stressor. Following exercise, $\mathrm{CD}^{+} 6^{+}$ monocytes are preferentially mobilized from the marginal pool where they are sequestered because of a high expression of adhesion molecules like very late antigen- 4 and CD11d [31-34]. These studies were performed before the nomenclature consensus in 2010 and only refer to 2 monocyte subpopulations $\left(\mathrm{CD} 14^{+} \mathrm{CD} 16^{+}\right.$and $\left.\mathrm{CD} 14^{+} \mathrm{CD} 16^{-}\right)$without further subdivision of the $\mathrm{CD}^{+} 6^{+}$cells. Later studies, allowing for the trichotomy of monocytes, offered more insight in Mon2 and Mon3 behaviour following exercise of moderate intensity [35] or a maximal exercise bout [16] in healthy volunteers.

Up to now, no data existed on the acute exercise-induced monocyte trafficking in $\mathrm{CKD}$ or $\mathrm{CHF}$, both conditions that benefit from exercise training programs in terms of lowering cardiovascular risk $[9,36]$. In the present study, a similar increase of Mon2 and Mon3 was confirmed in CKD and in HS. However, in CHF, the overall exercise-induced response of monocyte subsets was clearly blunted despite comparable exercise-related parameters as the CKD patients.

To investigate whether this could be related to a different chemotactic response, serum levels of IL-6 and MCP-1 were quantified before and immediately after peak exercise. $M C P-1$, a CCR2 ligand, plays an important role in monocyte mobilization and their selective recruitment into tissues [37]. Besides production by inflammatory cells and endothelial cells, MCP-1 is a contraction-regulated myokine with a possible role in the exercise-induced changes in the immune system [38]. Following exercise at moderate intensity, mRNA expression of MCP-1 is upregulated in skeletal muscle cells, coinciding with an increased serum concentration that is even more pronounced after high intensity exercise [39]. In this study, a short bout of strenuous exercise elicits a significant increase in MCP-1 in healthy subjects, whereas no change was observed in CKD or CHF patients. Hypothetically, this could be attributed to the higher observed levels at baseline, to a reduced vascular shear stress, to the short exercise duration (8-10 $\mathrm{min}$ ), or to a decreased muscle mass. Interleukin- 6 is another contraction-regulated myokine. The release of IL-6 from contracting skeletal muscle [14] may facilitate a broad anti-inflammatory response via effects on liver as well as on different leukocyte populations (reviewed in [40]). The magnitude of this effect is affected by the mode, intensity, and duration of exercise [41]. In this study, we detected only a small, nonsignificant increase in IL-6 following exercise, possibly because of the short duration of exercise. Nevertheless, the increase in IL-6 significantly correlated with the magnitude of total leukocyte increase. Taken together, a differentially regulated chemotactic response of MCP-1 or IL-6 is not the only explanation for the observed between-group variations in monocyte subsets following strenuous exercise.

Another explanation is that the blunted hemodynamic response during exercise in CHF (lower cardiac output, 
lower peak heart rate, and peak systolic blood pressure) is insufficient to recruit Mon2 and Mon3 that are avidly adhered to activated vascular endothelium. The observed strong association between peak heart rate, circulatory power, and $\mathrm{VO}_{2}$ peak supports this hypothesis. Release of $\mathrm{CD}^{+} 6^{+}$ monocytes into the circulation is known to be in part catecholamine dependent [31], but the strong relationship between circulatory power and $\mathrm{VO}_{2}$ peak and the response in monocyte subsets appeared to be independent of betablocker use. In conclusion, the release of Mon2 and Mon3 in the circulation, at the expense of the percentage Monl, is strongly driven by hemodynamic responses to exercise, which could explain the blunted response observed in CHF.

3.2.3. Limitations. In the present study, the effects of strenuous exercise were assessed 10 minutes after peak exercise. However, it is known that the exercise-induced effect on leukocytes follows a biphasic pattern, characterized by an immediate and delayed response (2-4 hours after exercise). In future studies, it would be interesting to investigate the time course of monocyte subsets over a longer time period.

\section{Conclusion}

Monocytes play a substantial role in systemic low-grade inflammation that is associated with cardiovascular disease, with distinct functions for the 3 monocyte subsets. Whereas the anti-inflammatory effect of exercise training is well established in chronic diseases such as $\mathrm{CKD}$ and $\mathrm{CHF}$, the response to acute exhaustive exercise is far less explored.

This study is the first to show that CKD patients, despite a lower exercise capacity and presence of low-grade inflammation, show a comparable acute exercise-induced change in monocyte subsets as healthy subjects. This effect is characterized by an increase in proangiogenic and proinflammatory Mon2 and Mon3, at the expense of Mon1. However, in CHF patients this effect is clearly attenuated and is strongly driven by a decreased hemodynamic response to exercise. Our findings offer more insight into the dynamic inflammatory response of acute exercise in different disease states, which is essential for the further unravelling of the mechanisms underlying the long-term beneficial effects of exercise training.

\section{Conflict of Interests}

The authors declare no conflict of interests.

\section{Acknowledgments}

The authors are very much indebted to Professor Viviane Conraads, former leader of our research group. She passed away on the December 12 last year, too early in her young life. We would like to thank Angelika Jürgens en Annemie Meessen for blood sampling, Bert Ectors and Professor Paul Beckers for performing cardiopulmonary exercise tests, and Dr. Guy De Schutter for assistance with recruiting the healthy subjects. The work was supported by the Center for Cell Therapy and Regenerative Medicine (Fund for Cell Therapy of the Antwerp University Hospital, Grant no. 21HMT05). Amaryllis H. Van Craenenbroeck is supported by a research grant from the University of Antwerp. Emeline M. Van Craenenbroeck is supported by the Research Foundation Flanders (FWO).

\section{References}

[1] A. M. Zawada, K. S. Rogacev, B. Rotter et al., "SuperSAGE evidence for $\mathrm{CD} 14^{++} \mathrm{CD} 16^{+}$monocytes as a third monocyte subset," Blood, vol. 118, no. 12, pp. e50-e61, 2011.

[2] L. Ziegler-Heitbrock, P. Ancuta, S. Crowe et al., "Nomenclature of monocytes and dendritic cells in blood," Blood, vol. 116, no. 16, pp. e74-e80, 2010.

[3] E. Shantsila, B. Wrigley, L. Tapp et al., "Immunophenotypic characterization of human monocyte subsets: possible implications for cardiovascular disease pathophysiology," Journal of Thrombosis and Haemostasis, vol. 9, no. 5, pp. 1056-1066, 2011.

[4] B. J. Wrigley, G. Y. Lip, and E. Shantsila, "The role of monocytes and inflammation in the pathophysiology of heart failure," European Journal of Heart Failure, vol. 13, no. 11, pp. 1161-1171, 2011.

[5] A. Tedgui and Z. Mallat, "Cytokines in atherosclerosis: pathogenic and regulatory pathways," Physiological Reviews, vol. 86, no. 2, pp. 515-581, 2006.

[6] R. T. Gansevoort, R. Correa-Rotter, B. R. Hemmelgarn et al., "Chronic kidney disease and cardiovascular risk: epidemiology, mechanisms, and prevention," The Lancet, vol. 382, no. 9889, pp. 339-352, 2013.

[7] V. M. Conraads, J. M. Bosmans, and C. J. Vrints, "Chronic heart failure: an example of a systemic chronic inflammatory disease resulting in cachexia," International Journal of Cardiology, vol. 85, no. 1, pp. 33-49, 2002.

[8] U. G. Bronas, "Exercise training and reduction of cardiovascular disease risk factors in patients with chronic kidney disease," Advances in Chronic Kidney Disease, vol. 16, no. 6, pp. 449-458, 2009.

[9] C. M. O’Connor, D. J. Whellan, K. L. Lee et al., "Efficacy and safety of exercise training in patients with chronic heart failure HF-ACTION randomized controlled trial," The Journal of the American Medical Association, vol. 301, no. 14, pp. 1439-1450, 2009.

[10] S. Adamopoulos, J. Parissis, C. Kroupis et al., "Physical training reduces peripheral markers of inflammation in patients with chronic heart failure," European Heart Journal, vol. 22, no. 9, pp. 791-797, 2001.

[11] J. L. Viana, G. C. Kosmadakis, E. L. Watson et al., "Evidence for anti-inflammatory effects of exercise in CKD," Journal of the American Society of Nephrology, 2014.

[12] H. Gabriel, A. Urhausen, L. Brechtel, H.-J. Muller, and W. Kindermann, "Alterations of regular and mature monocytes are distinct, and dependent of intensity and duration of exercise," European Journal of Applied Physiology and Occupational Physiology, vol. 69, no. 2, pp. 179-181, 1994.

[13] M. J. Hubal, T. C. Chen, P. D. Thompson, and P. M. Clarkson, "Inflammatory gene changes associated with the repeatedbout effect," The American Journal of Physiology-Regulatory Integrative and Comparative Physiology, vol. 294, no. 5, pp. R1628-R1637, 2008.

[14] A. Steensberg, G. van Hall, T. Osada, M. Sacchetti, B. Saltin, and B. K. Pedersen, "Production of interleukin-6 in contracting 
human skeletal muscles can account for the exercise-induced increase in plasma interleukin-6," The Journal of Physiology, vol. 529, part 1, pp. 237-242, 2000.

[15] U. R. Mikkelsen, C. Couppé, A. Karlsen et al., "Life-long endurance exercise in humans: circulating levels of inflammatory markers and leg muscle size," Mechanisms of Ageing and Development, vol. 134, no. 11-12, pp. 531-540, 2014.

[16] E. Shantsila, L. D. Tapp, B. J. Wrigley et al., "The effects of exercise and diurnal variation on monocyte subsets and monocyteplatelet aggregates," European Journal of Clinical Investigation, vol. 42, no. 8, pp. 832-839, 2012.

[17] J. E. Hansen, D. Y. Sue, and K. Wasserman, "Predicted values for clinical exercise testing," The American Review of Respiratory Disease, vol. 129, no. 2, pp. S49-S55, 1984.

[18] A. S. Levey, L. A. Stevens, C. H. Schmid et al., "A new equation to estimate glomerular filtration rate," Annals of Internal Medicine, vol. 150, no. 9, pp. 604-612, 2009.

[19] A. Schlitt, G. H. Heine, S. Blankenberg et al., "CD14 ${ }^{+} \mathrm{CD} 16^{+}$ monocytes in coronary artery disease and their relationship to serum TNF- $\alpha$ levels," Thrombosis and Haemostasis, vol. 92, no. 2, pp. 419-424, 2004.

[20] M. A. Venneri, M. de Palma, M. Ponzoni et al., "Identification of proangiogenic TIE2-expressing monocytes (TEMs) in human peripheral blood and cancer," Blood, vol. 109, no. 12, pp. 52765285, 2007.

[21] S. Fujiyama, K. Amano, K. Uehira et al., "Bone marrow monocyte lineage cells adhere on injured endothelium in a monocyte chemoattractant protein-1-dependent manner and accelerate reendothelialization as endothelial progenitor cells," Circulation Research, vol. 93, no. 10, pp. 980-989, 2003.

[22] K. L. Wong, J. J.-Y. Tai, W.-C. Wong et al., "Gene expression profiling reveals the defining features of the classical, intermediate, and nonclassical human monocyte subsets," Blood, vol. 118, no. 5, pp. e16-e31, 2011.

[23] K. S. Rogacev, S. Seiler, A. M. Zawada et al., "CD14 ${ }^{++} \mathrm{CD} 16^{+}$ monocytes and cardiovascular outcome in patients with chronic kidney disease," European Heart Journal, vol. 32, no. 1, pp. 8492, 2011

[24] K. S. Rogacev, B. Cremers, A. M. Zawada et al., "CD14 ${ }^{++} \mathrm{CD} 16^{+}$ monocytes independently predict cardiovascular events: a cohort study of 951 patients referred for elective coronary angiography," Journal of the American College of Cardiology, vol. 60, no. 16, pp. 1512-1520, 2012.

[25] B. J. Wrigley, E. Shantsila, L. D. Tapp, and G. Y. H. Lip, "CD14 ${ }^{++} \mathrm{CD} 16^{+}$monocytes in patients with acute ischaemic heart failure," European Journal of Clinical Investigation, vol. 43, no. 2, pp. 121-130, 2013.

[26] C. Barisione, S. Garibaldi, G. Ghigliotti et al., "CD14CD16 monocyte subset levels in heart failure patients," Disease Markers, vol. 28, no. 2, pp. 115-124, 2010.

[27] O. Amir, I. Spivak, I. Lavi, and M. A. Rahat, "Changes in the monocytic subsets $\mathrm{CD} 14^{\mathrm{dim}} \mathrm{CD} 16^{+}$and $\mathrm{CD} 14^{++} \mathrm{CD} 16^{-}$in chronic systolic heart failure patients," Mediators of Inflammation, vol. 2012, Article ID 616384, 9 pages, 2012.

[28] A. M. Zawada, K. S. Rogacev, S. H. Schirmer et al., "Monocyte heterogeneity in human cardiovascular disease," Immunobiology, vol. 217, no. 12, pp. 1273-1284, 2012.

[29] M. Vaduganathan, S. J. Greene, J. Butler et al., "The immunological axis in heart failure: importance of the leukocyte differential," Heart Failure Reviews, vol. 18, no. 6, pp. 835-845, 2013.
[30] J. G. Wheeler, M. E. Mussolino, R. F. Gillum, and J. Danesh, "Associations between differential leucocyte count and incident coronary heart disease: 1764 incident cases from seven prospective studies of 30374 individuals," European Heart Journal, vol. 25, no. 15, pp. 1287-1292, 2004.

[31] B. Steppich, F. Dayyani, R. Gruber, R. Lorenz, M. Mack, and H. W. L. Ziegler-Heitbrock, "Selective mobilization of $\mathrm{CD} 14^{+}$ $\mathrm{CD}^{+} 6^{+}$monocytes by exercise," The American Journal of Physiology-Cell Physiology, vol. 279, no. 3, pp. C578-C586, 2000.

[32] I. Heimbeck, T. P. J. Hofer, C. Eder et al., "Standardized singleplatform assay for human monocyte subpopulations: lower $\mathrm{CD} 14^{+} \mathrm{CD} 16^{++}$monocytes in females," Cytometry A, vol. 77, no. 9, pp. 823-830, 2010.

[33] R. J. Simpson, B. K. McFarlin, C. McSporran, G. Spielmann, B. Ó. Hartaigh, and K. Guy, "Toll-like receptor expression on classic and pro-inflammatory blood monocytes after acute exercise in humans," Brain, Behavior, and Immunity, vol. 23, no. 2, pp. 232-239, 2009.

[34] P. Ancuta, R. Rao, A. Moses et al., "Fractalkine preferentially mediates arrest and migration of $\mathrm{CD}^{+} 6^{+}$monocytes," The Journal of Experimental Medicine, vol. 197, no. 12, pp. 1701-1707, 2003.

[35] S. Hong and P. J. Mills, "Effects of an exercise challenge on mobilization and surface marker expression of monocyte subsets in individuals with normal versus elevated blood pressure," Brain, Behavior, and Immunity, vol. 22, no. 4, pp. 590-599, 2008.

[36] S. Heiwe and S. H. Jacobson, "Exercise training for adults with chronic kidney disease," Cochrane Database of Systematic Reviews, no. 10, Article ID CD003236, 2011.

[37] F. B. Mayr, A. O. Spiel, J. M. Leitner et al., "Influence of the Duffy antigen on pharmacokinetics and pharmacodynamics of recombinant monocyte chemoattractant protein (MCP-1, CCL2) in vivo," International Journal of Immunopathology and Pharmacology, vol. 22, no. 3, pp. 615-625, 2009.

[38] S. Raschke and J. Eckel, "Adipo-Myokines: two sides of the same coin-mediators of inflammation and mediators of exercise," Mediators of Inflammation, vol. 2013, Article ID 320724, 16 pages, 2013.

[39] J. M. Peake, K. Suzuki, M. Hordern, G. Wilson, K. Nosaka, and J. S. Coombes, "Plasma cytokine changes in relation to exercise intensity and muscle damage," European Journal of Applied Physiology, vol. 95, no. 5-6, pp. 514-521, 2005.

[40] M. Gleeson, N. C. Bishop, D. J. Stensel, M. R. Lindley, S. S. Mastana, and M. A. Nimmo, "The anti-inflammatory effects of exercise: mechanisms and implications for the prevention and treatment of disease," Nature Reviews Immunology, vol. 11, no. 9, pp. 607-615, 2011.

[41] C. P. Fischer, "Interleukin-6 in acute exercise and training: what is the biological relevance?" Exercise Immunology Review, vol. 12, pp. 6-33, 2006. 


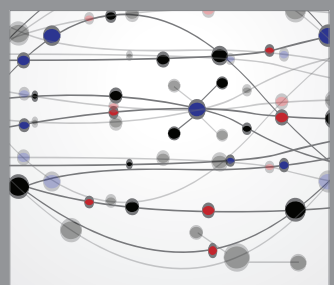

The Scientific World Journal
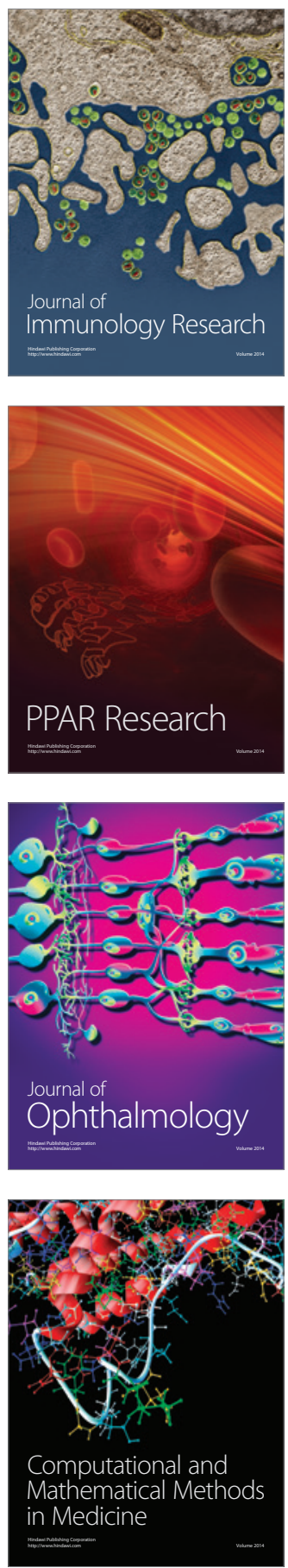

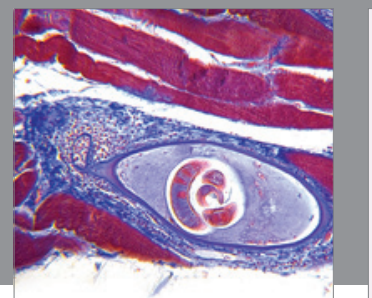

Gastroenterology

Research and Practice
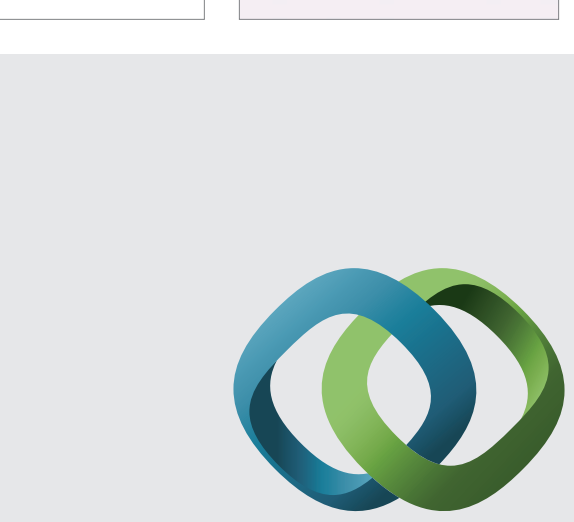

\section{Hindawi}

Submit your manuscripts at

http://www.hindawi.com
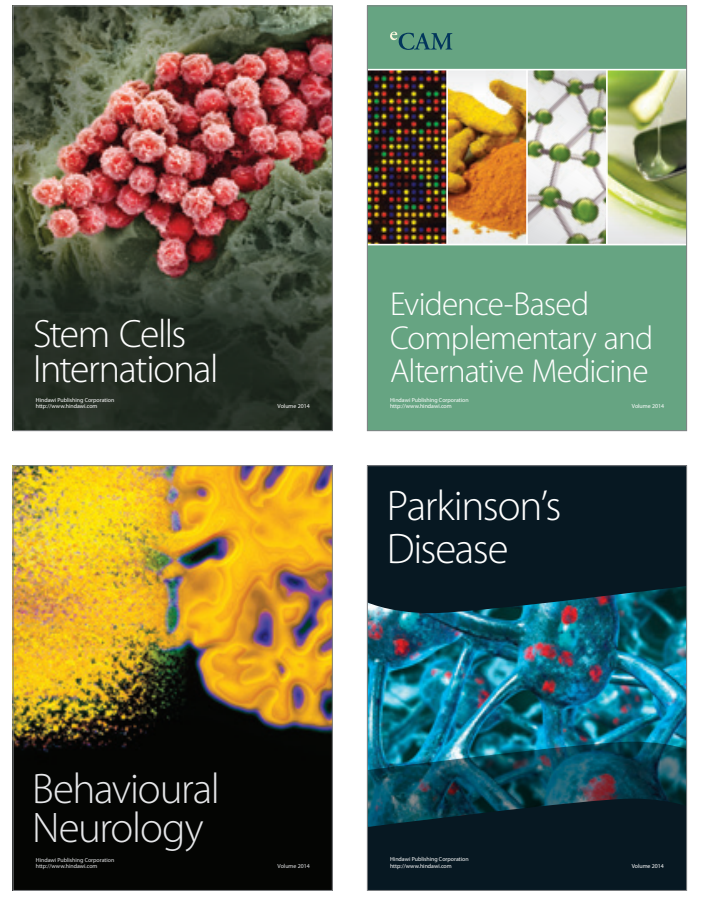
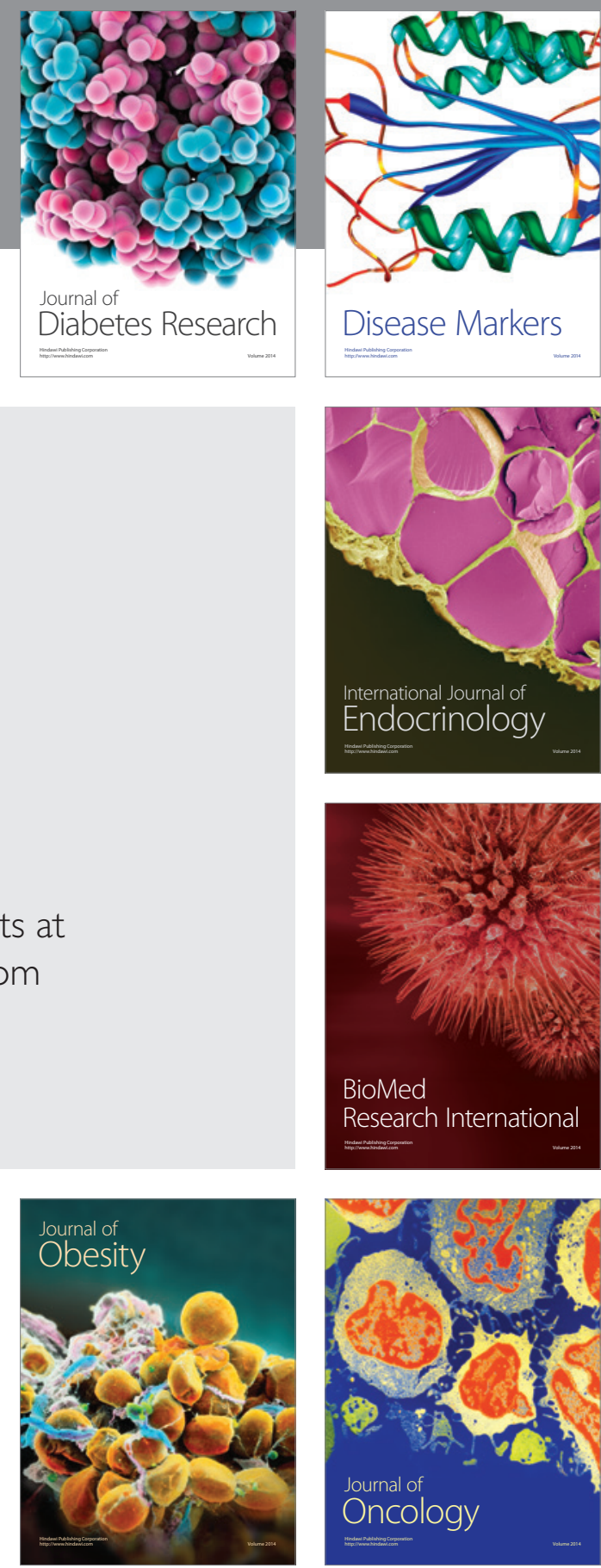

Disease Markers
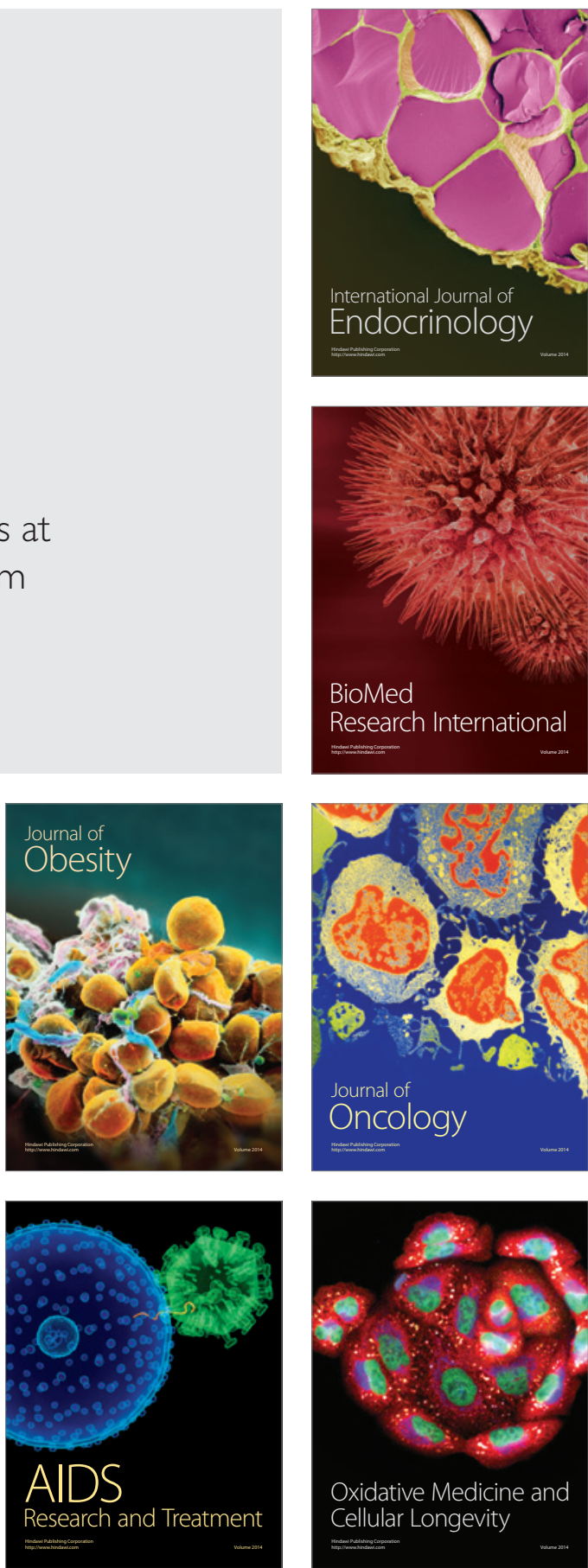